\title{
Análisis cualitativo de los determinantes de la innovación en una economía emergente
}

\section{Qualitative approach about innovation determinants in an emerging economy}

Dr. Javier Del Carpio-Gallegos es Decano de la Facultad de Ingeniería de la Universidad ESAN (Perú) (jdelcarpio@esan.edu. pe) (https://orcid.org/0000-0001-6050-5754)

Dr. Francesc Miralles es Academic Dean en La Salle - Universidad Ramon Llull y Director del Innova Insitute (España) (francesc.miralles@salle.url.edu) (https://orcid.org/0000-0002-5251-5423)

\section{Resumen}

Las empresas de manufactura se esfuerzan por ser innovadoras y, de esta manera, ser competitivas en el mercado. Estas empresas son muy importantes en una economía emergente debido a su contribución al Producto Interno Bruto y a la generación de puestos de trabajo para la población. Por ello, el objetivo de este estudio es identificar cuáles son los factores internos y externos que mejoran la capacidad de innovación de las empresas peruanas de manufactura y, a su vez, favorecen su competitividad. Para ello, se aplicó un enfoque cualitativo basado en el «modelo de las seis C» de Glaser, que forma parte de la teoría fundamentada, y se entrevistó a gerentes de las empresas de manufactura peruanas. A partir del análisis y procesamiento de estos datos con el software de análisis cualitativo (QDA) Atlas ti®, se encontró que los proveedores y los clientes proporcionan valiosa información para innovar, y que la aplicación del diseño y la adquisición de maquinaria favorecen las innovaciones de estas empresas. En esa medida, el presente estudio contribuye a identificar aquellos factores que ayudan a las empresas de manufactura peruanas a mejorar su capacidad de innovación. Así, los gerentes de las empresas de manufactura deben identificar aquellos factores que favorecen la implementación de innovaciones y, de esta manera, lograrán que sus empresas sean más competitivas.

\begin{abstract}
Manufacturing companies strive to be innovative and competitive. These companies are very important in an emerging economy due to their contribution to a country's gross domestic product and the generation of jobs. Therefore, the objective of this article is to identify the internal and external factors that improve the innovation capacity of Peruvian manufacturing companies and, in turn, favor their competitiveness. A qualitative approach was applied based on the Glaser "six-C model», which is part of the grounded theory; for this, the managers of the Peruvian manufacturing companies were interviewed. From the analysis

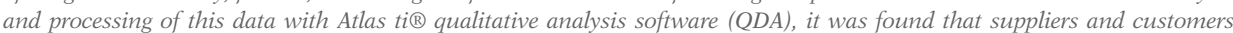
provide valuable information to innovate; additionally, the application of the design and the acquisition of machinery favor the innovations of these companies. To that extent, this qualitative study contributes to identify those factors that help Peruvian manufacturing companies improve their innovation capacity. Thus, manufacturing companies' managers must identify those factors that favor the implementation of innovations to make their companies more competitive.
\end{abstract}

\section{Palabras clave I keywords}

Innovación, análisis cualitativo, manufactura, economía emergente, desarrollo tecnológico, teoría fundamentada. Innovation, qualitative analysis, manufacturing, emerging economies, technological development, grounded theory.

Cómo citar: Del Carpio-Gallegos, J. y Miralles, F. (2019). Análisis cualitativo de los determinantes de la innovación en una economía emergente. Retos Revista de Ciencias de la Administración y Economía, 9(17), 161-175. https://doi.org/10.17163/ret.n17.2019.10 


\section{Introducción}

La innovación se ha convertido en un tema de mucho interés para las empresas debido a los resultados que obtienen las que la implementan. Así, en algunos casos, crean una ventaja competitiva (Herman, Hady \& Arafah, 2018) con su desempeño innovador (Martínez-Costa, Jimenez-Jimenez \& Castro-del-Rosario, 2018). Una revisión de la literatura muestra que los estudios sobre innovación en las economías desarrolladas son principalmente cuantitativos (Ketata, Sofka \& Grimpe, 2015). También, hay un número importante de aplicaciones cuantitativas en investigaciones sobre innovación en países latinoamericanos, como es el caso de Colombia (v.gr. Albis \& Álvarez, 2017). Estos hallazgos muestran poca atención a investigaciones con enfoques cualitativos en las economías latinoamericanas, en el que se puede citar el caso de Brasil (v.gr. Ferreira de Lara \& Neves Guimarães, 2014).

Se debe tomar en cuenta que las economías latinoamericanas pasaron por un periodo de bonanza económica, para luego enfrentar una realidad distinta, en la que destaca la baja estabilidad política (Olavarrieta \& Villena, 2014), alto niveles de corrupción (Paunov, 2016), y de informalidad (Heredia, Flores, Geldes \& Heredia, 2017). Es este contexto el que se convierte, en algunos casos, en un limitante para llevar a cabo las innovaciones.

El presente estudio se ha centrado en las empresas de manufactura peruanas, debido a que este sector económico tiene un alto impacto en la economía del país suramericano. De hecho, el sector de manufactura representa más del $16 \%$ de la producción nacional, según el Instituto Nacional de Estadística e Informática (INEI, 2018). Por lo tanto, se busca identificar cuáles son los factores internos y externos que mejoran la capacidad de innovación de estas empresas. De esta manera, se contribuye con la literatura sobre la innovación en el sector de manufactura de una economía emergente. En esta línea, el artículo está organizado en cuatro partes. En primer lugar, se presenta la revisión de literatura y se señala cómo la literatura identifica los factores externos e internos que favorecen la realización de innovación. En segundo lugar, se describen los materiales y método empleados, detallándose los principios del modelo de las «seis C de Glaser», cómo se procedió con la selección de la muestra, la selección de los participantes, los métodos de recolección de la información y se presentan los datos arrojados. En tercer lugar, se presentan los resultados según la estructura de las seis C de Glaser, para finalmente presentar la discusión y las conclusiones.

\subsection{Revisión de la literatura}

\subsubsection{Contexto y condiciones de una economía emergente}

Cuando se analiza el contexto de una economía emergente como la peruana, se pueden considerar dos aspectos: por un lado, el interés por realizar investigaciones sobre las condiciones en las cuales las empresas llevan a cabo innovaciones y, por otro lado, los retos y dificultades que estas empresas enfrentan. En relación a las investigaciones realizadas Castellacci \& Natera (2012) señalan que se han realizado numerosas investigaciones cuantitativas; sin embargo, todavía se deben realizar futuras investigaciones, en particular con enfoques y diseños cualitativos que permitan profundizar 
los hallazgos. Se sabe que las empresas que quieren mejorar su capacidad de innovación deben establecer vinculaciones con sus proveedores, clientes, laboratorios, universidades, entre otros (Ferraris, Santoro \& Dezi, 2017).

En tanto, desde el punto de vista de los retos y dificultades, se pueden mencionar dos estudios importantes. El primero es de Sanz \& Jones (2013), quienes afirman que los países latinoamericanos hace más de una década han pasado por una etapa de bonanza económica que les ha permitido reducir los niveles de pobreza y el crecimiento de una clase media, debido a la mejora de los precios de los minerales. El segundo estudio de Olavarrieta \& Villena (2014) considera que las empresas latinoamericanas que desarrollan innovación enfrentan retos y dificultades, como la carencia de políticas que alienten el desarrollo de las innovaciones.

No resulta baladí hacer algunas precisiones en relación a términos como la «intensidad tecnológica», que según Zawislak, Fracasso \& Tello-Gamarra (2018) se obtiene dividiendo los gastos en Investigación y Desarrollo (I+D) entre los activos o las ventas. Para la Organización para la Cooperación y el Desarrollo Económicos (OECD, 2011), las empresas según su intensidad tecnológica se clasifican en baja, baja-media, media-alta y alta intensidad tecnológica.

Por otro lado, Kim, Park \& Paik (2018) indican que la capacidad de innovación de la empresa, que es el potencial para crear innovaciones, puede ser analizada en varias dimensiones, que van en función de los diferentes tipos de innovación: producto, proceso, organizacional o de marketing.

\subsubsection{Factores internos}

Los factores internos, según Lee, Leong, Hew \& Ooi (2013) son las variables internas que están bajo el control de la empresa y que le permiten mejorar su capacidad de innovación. Estas variables son el diseño e ingeniería industrial, la adquisición de maquinaria, la certificación y control de calidad, y la capacitación del personal.

El diseño hace referencia a una forma importante de transferir las ideas o conocimientos (Simeone, Secundo, \& Schiuma, 2017). Además, éste ayuda de manera significativa en el desarrollo de nuevos productos. En el caso de las empresas de manufactura peruanas, Tello (2017) encontró que éstas mejoran su capacidad de innovación mediante la adquisición de maquinaria. Por su parte, el estándar de calidad ISO 9000 se basa en la definición de conformidad de calidad para asegurar a los clientes que un producto o servicio de calidad será suministrado de manera consistente (Bourke \& Roper, 2017).

En la gestión de la innovación, la motivación de los profesionales juega un papel crucial en los procesos de creación colaborativa de conocimientos por contar con mayores competencias (Papa et al., 2018).

\subsubsection{Factores externos}

Roper, Love \& Bonner (2017) afirman que parte del proceso de innovación realizado por las empresas consiste en recolectar información certera de una variedad de fuentes externas a la empresa, tales como clientes, proveedores, universidades, entre otros, las cuales contribuyen a reducir la incertidumbre en las probabilidades de innovar. En esta línea, Saldanha, Mithas \& Krishnan, (2017) señalan que los clientes 
son una fuente de conocimiento que ayuda a las empresas mediante la modificación de productos existentes para un mejor uso; por ello, se hace necesario involucrarlos en los procesos de innovación de la empresa.

Por su parte, los proveedores juegan un rol importante en el proceso de innovación de la empresa. En tal sentido, una fuerte relación entre proveedores y empresa permite alcanzar un adecuado ambiente de innovación mediante la mejora de la calidad del producto y manejo adecuado de costos (Jajja et al., 2017).

Las empresas enfrentan a los competidores aplicando distintas estrategias. En algunos casos, imitan productos nuevos ofrecidos a precios bajos y, en otros contextos, ofrecen a sus consumidores productos diferenciados, tratando de generar lealtad en los clientes (Liu \& Atuahene-Gima, 2018). En este sentido, las organizaciones implementan procesos de innovación, que se ven influenciados por empresas e individuos; en ese proceso, el rol de los consultores es de suma importancia debido a su conocimiento y experiencia (Musiolik et al., 2018).

\section{Materiales y métodos}

Para propósitos de esta investigación, se decidió emplear el «modelo de las seis C» de Glaser (Gandomani et al., 2013) como herramienta metodológica para la obtención de información primaria acerca de los principales factores que impactan en el desarrollo de la innovación en empresas de manufactura. El modelo propuesto forma parte de la teoría fundamentada, que permite a los investigadores desarrollar la teoría a través de la información recolectada y favorece una constante retroalimentación con la problemática que se estudia (Strauss \& Corbin, 1994).

Se ha preferido utilizar la teoría fundamentada porque la innovación es un proceso de interacción entre diferentes variables y actores, tanto internos como externos, que permiten a la empresa acumular conocimiento para convertirlo en productos o procesos innovadores. Además, se ha preferido usar, como instrumento, una guía de entrevista semi-estructurada porque permite al entrevistador mencionar variables o situaciones que van más allá de las preguntas. De esta manera se pueden reunir datos que permitan identificar una teoría. Entonces, mediante el uso de estas entrevistas a personal de empresas del sector y el contraste de la información obtenida con la literatura correspondiente, se comienza a generar un patrón de comportamiento para cada factor, el cual será nuestra base para la investigación. Aunque la teoría fundamentada no implica formular el problema de investigación desde el principio en base a una revisión importante de la literatura, la aplicación no está prohibida (Dunne, 2011).

Para la codificación teórica, se empleó el «modelo de las seis C» de Glaser (Gandomani et al., 2013), como último paso del estudio. Este modelo ayuda al investigador a buscar conexiones y relaciones entre las categorías centrales y otras que emergieron. A continuación, se muestra la terminología utilizada por el precitado modelo: 
[...] contexto (el lugar donde la categoría está en juego), condición (un factor que es un prerrequisito para que la categoría surja), causa (una razón para que la categoría ocurra), consecuencia (un resultado o efecto de la ocurrencia de la categoría), contingencia (un factor moderador entre las categorías y las consecuencias), y covarianza (las categorías que pueden variar la una con la otra) (Van Waardenburg \& Van Vliet, 2013, p. 2158).

Los entrevistados, en su mayoría, se desempeñan como gerentes generales, jefes de área y personal designado de empresas representativas del sector. Además, la mayoría de ellos representan a empresas textiles y de alimentos (40\%). A continuación (tabla 1), se presenta la relación de las empresas objeto de estudio y se indica el giro del negocio, la intensidad tecnológica, el tamaño y edad de la empresa.

Tabla 1. Relación de empresas seleccionadas

\begin{tabular}{|l|l|l|l|}
\hline Empresa & \multicolumn{1}{|c|}{ Industrial } & \multicolumn{1}{|c|}{$\begin{array}{c}\text { Intensidad } \\
\text { Tecnológica }\end{array}$} & $\begin{array}{l}\text { Tamaño de } \\
\text { la empresa }\end{array}$ \\
\hline E01 & Alimentos & Baja & Mediana \\
\hline E02 & Alimentos & Baja & Pequeña \\
\hline E03 & Alimentos & Baja & Pequeña \\
\hline E04 & Textiles & Baja & Pequeña \\
\hline E05 & Textiles & Baja & Grande \\
\hline E06 & Textiles & Baja & Mediana \\
\hline E07 & $\begin{array}{l}\text { Fabricación de productos de caucho y } \\
\text { plástico }\end{array}$ & Baja-media & Pequeña \\
\hline E08 & $\begin{array}{l}\text { Fabricación de productos de caucho y } \\
\text { plástico }\end{array}$ & Baja-media & Mediana \\
\hline E09 & Fabricación de vehículos automotores & Media-alta & Pequeña \\
\hline E10 & Fabricación de vehículos automotores & Media-alta & Pequeña \\
\hline E11 & Fabricación de vehículos automotores & Media-alta & Pequeña \\
\hline E12 & $\begin{array}{l}\text { Fabricación de productos elaborados de } \\
\text { metal }\end{array}$ & Baja-media & Grande \\
\hline E13 & $\begin{array}{l}\text { Fabricación de productos elaborados de } \\
\text { metal }\end{array}$ & $\begin{array}{l}\text { Baja-media } \\
\text { micos })\end{array}$ & Mediana \\
\hline E14 & Fabricación de productos de cuero & Baja & Pequeña \\
\hline E15 & Fabricación de maquinaria y equipo & Media-alta & Pequeña \\
\hline E16 & Grande \\
\hline
\end{tabular}

Algunas entrevistas fueron grabadas digitalmente, mientras que otras fueron respondidas por correo electrónico. Se empezó la recolección el 1 de febrero de 2017. Cabe resaltar que, al finalizar ambas, cuando se observó que existían omisiones en las respuestas de algunas preguntas, se decidió volver a entrevistar a estas personas. Posteriormente, se transcribió toda la grabación mediante el procesador de texto Microsoft Word®. Se trabajó con un total de 97 códigos, que se agruparon en 2 categorías (tabla 2). 
Tabla 2. Cantidad de citas de factores internos y externos

\begin{tabular}{|l|c|}
\hline \multicolumn{1}{|c|}{ Categorías } & $f$ \\
\hline \multicolumn{2}{|c|}{ Factores internos } \\
\hline Diseño e ingeniería industrial & 13 \\
\hline Adquisición de maquinaria & 7 \\
\hline Certificación y control de calidad & 5 \\
\hline Capacitación de personal Factores externos & 5 \\
\hline Adquisición de software & 4 \\
\hline Personal capacitado & 3 \\
\hline Estudio de mercado & 3 \\
\hline Total & 40 \\
\hline & 16 \\
\hline Clientes & 13 \\
\hline Proveedores & 12 \\
\hline Competencia & 7 \\
\hline Consultores externos & 5 \\
\hline Universidades & 3 \\
\hline Asociación de empresas & 1 \\
\hline Institutos de investigación gubernamentales & 57 \\
\hline Total & 97 \\
\hline Total de códigos & \\
\hline
\end{tabular}

Nota: Para la codificación, el análisis y la elaboración de redes, se usó el software de análisis cualitativo (QDA) Atlas ti®. El análisis final de los resultados se completó el 31 de mayo de 2018.

La parte del análisis se compone de clasificación, comparación, ponderación y la combinación de los datos obtenidos mediante las entrevistas, de manera que se extrajeran significados e implicaciones para revelar patrones o realizar una narrativa coherente. El modelo de análisis consiste en dos partes. En la primera parte, se prepararon las transcripciones, y se encontraron, refinaron y elaboraron conceptos, temas y eventos. Luego, se codificaron las entrevistas para esclarecer lo que el entrevistado ha dicho acerca de los conceptos identificados, temas y eventos. En la segunda parte, se siguieron varios caminos: Se compararon conceptos y temas a lo largo de las entrevistas o se combinar eventos separados para formular una descripción del medio. De esta manera, se buscaba responder a las preguntas de investigación para sacar conclusiones teóricas generales.

\section{Análisis y resultados}

Los resultados son presentados, a continuación, a partir de la aplicación del «modelo de las seis C» de Glaser y el uso del software de análisis cualitativo (QDA) Atlas ti®. 


\subsection{Aplicación del modelo de las seis C de Glaser}

En la figura 1, se muestra el «modelo de las seis C» de Glaser (Gandomani et al., 2015), y que se utilizará para presentar los resultados del presente estudio.

Figura 1. Modelo de las seis $\mathrm{C}$ de Glasser

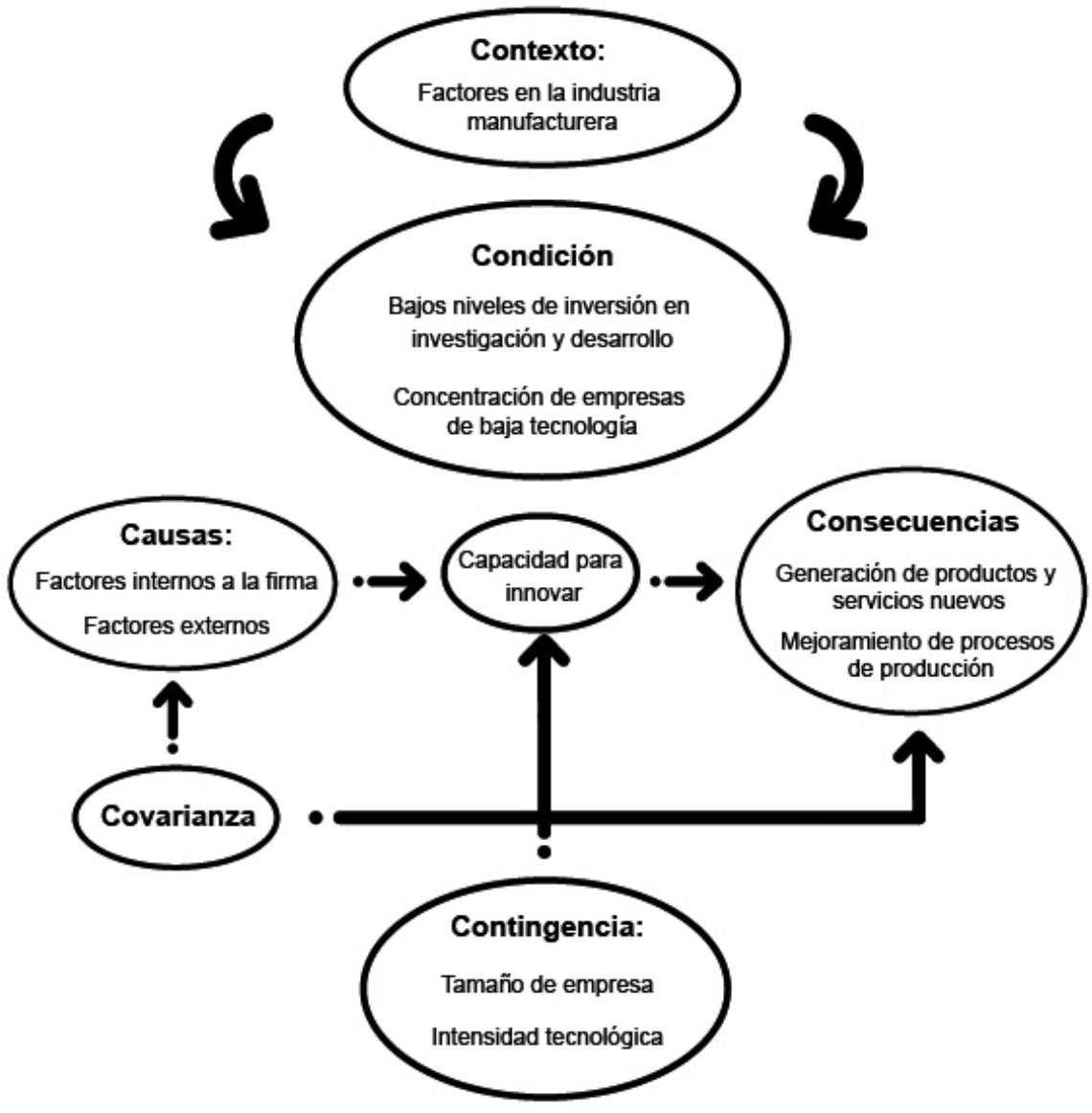

Fuente: Gandomani et al. (2015, p. 300)

\subsection{Contexto}

Hace quince años, la economía peruana mostró un crecimiento sostenido (Scott \& Chaston, 2012), que la convirtió en una de las economías de mayor crecimiento en la región. Sin embargo, después de la crisis de los commodities (Brenes et al., 2016) se obligó a las empresas a enfrentar un cambio de realidad. A pesar de esta situación, las empresas peruanas tienden a invertir muy poco en Investigación y Desarrollo (I+D), 
y prefieren innovar comprando maquinaria, hardware y software (Tello, 2017). También enfrentan competencia informal (Heredia et al., 2017) y tienen problemas para obtener recursos financieros para promover la innovación (Pérez et al., 2018).

\subsection{Condiciones}

El Ministerio de la Producción (2016), en su estudio de la situación actual de la innovación en la industria manufacturera, señaló que "la manufactura es uno de los sectores que tienen mayor participación en el Producto Interno Bruto, llegando a $13.5 \%$ en el 2015" (ob. cit.,p.15). También indicó que para 2014, “más del $95 \%$ de las empresas manufactureras son nacionales y en su mayoría pequeñas empresas” (ob. cit., p. 16).

\subsection{Causas}

La tabla 3 muestra ejemplos representativos de las respuestas que se obtuvieron en relación a los factores internos que alientan el desarrollo de la capacidad de innovación de las empresas:

Tabla 3. Respuestas de los entrevistados sobre factores internos

\begin{tabular}{|l|l|}
\hline $\begin{array}{c}\text { Descripción del tipo } \\
\text { de factores internos }\end{array}$ & \multicolumn{1}{c|}{ Ejemplo de citas } \\
\hline $\begin{array}{l}\text { Diseño e ingeniería indus- } \\
\text { trial }\end{array}$ & $\begin{array}{l}\text { "En el rubro del calzado, la evolución es casi constante, pues } \\
\text { la tecnología manifiesta notoriamente su aporte. Más allá de } \\
\text { aumentar la productividad, aumenta la calidad". (Fabricación } \\
\text { de productos de cuero, empresa 15) }\end{array}$ \\
\hline Adquisición demaquinaria & $\begin{array}{l}\text { "La adquisición de maquinaria, que involucra incorporación } \\
\text { de maquinarias y herramientas para mejoras del proceso y así } \\
\text { lograr optimizar todos los recursos". (Elaboración de cerámi- } \\
\text { cos, empresa 14) }\end{array}$ \\
\hline $\begin{array}{l}\text { Certificación y } \\
\text { control de calidad }\end{array}$ & $\begin{array}{l}\text { "No todas las empresas aplican prácticas de control de calidad } \\
\text { en todas las áreas. Por ello, no se mantienen y muchas fraca- } \\
\text { san con el pasar del tiempo... tenemos un área de innovación } \\
\text { y gestión que ayuda a mantener las ISO actualizadas". (Indus- } \\
\text { trias básicas de hierro y acero, empresa 12) }\end{array}$ \\
\hline Capacitación del personal & $\begin{array}{l}\text { "Para adquirir conocimientos en la vida laboral y profesional, } \\
\text { mejorando aptitudes y habilidades". (Fabricación de produc- } \\
\text { tos metálicos, empresa 12) }\end{array}$ \\
\hline
\end{tabular}

Las empresas de manufactura de baja y baja-media intensidad tecnológica desarrollan actividades de diseño e ingeniería industrial, adquisición de maquinaria, certificación y control de calidad, y la capacitación del personal. De esta manera, logran mejorar su capacidad de innovación. Estas actividades son realizadas independientemente del tamaño de la empresa. La tabla 4 muestra respuestas representativas que se obtuvieron en relación a los factores externos que alientan el desarrollo de la capacidad de innovación de las empresas: 
Tabla 4. Respuesta de los entrevistados sobre factores externos

\begin{tabular}{|l|l|}
\hline $\begin{array}{l}\text { Descripción del tipo } \\
\text { de factores externos }\end{array}$ & \multicolumn{1}{c|}{ Ejemplo de citas } \\
\hline $\begin{array}{l}\text { Consumidores y/o } \\
\text { Clientes }\end{array}$ & $\begin{array}{l}{[\ldots] \text { "los clientes nos ayudan mucho a saber si vamos en la dirección }} \\
\text { correcta con cada cambio hecho en la empresa, tanto en procesos } \\
\text { como en productos" (Elaboración de otros productos alimenticios, } \\
\text { empresa 3) }\end{array}$ \\
\hline Proveedores & $\begin{array}{l}{[\ldots] \text { "la retroalimentación con los proveedores que te mantienen al }} \\
\text { tanto de mejoras en sus productos y los clientes que te impulsan a } \\
\text { la mejora continua" (Otras industrias manufactureras, empresa 14) }\end{array}$ \\
\hline Competencia & $\begin{array}{l}\text { "Se realiza constante benchmarking con empresas competidoras, } \\
\text { analizando sus estrategias de venta" (Preparación de fibras textiles, } \\
\text { gran empresa, empresa 5) }\end{array}$ \\
\hline Consultores externos & $\begin{array}{l}\text { "Adicionalmente la empresa tiene en la visión la expansión en las } \\
\text { unidades de negocio, para lo cual se está trabajando en actividades } \\
\text { relacionadas a I+D, tanto interna como externa, con ayuda de con- } \\
\text { sultores externos" (Preparación de fibras textiles, empresa 5) }\end{array}$ \\
\hline
\end{tabular}

Las empresas de manufactura de baja y baja-media intensidad tecnológica tienden a vincularse con los consumidores, clientes, proveedores y consultores para obtener información o conocimiento que les permita mejorar su capacidad de innovación. Alguna de estas empresas ven a la competencia como un referente que las alienta a ser más competitivas. Por último, tenemos, en la tabla 5, las respuestas de las distintas empresas sobre la innovación:

Tabla 5. Respuesta de los entrevistados sobre innovación

\begin{tabular}{|l|l|}
\hline $\begin{array}{c}\text { Descripción de } \\
\text { tipo de innovación }\end{array}$ & \multicolumn{1}{c|}{ Ejemplo de citas } \\
\hline $\begin{array}{l}\text { Innovació } \\
\text { en producto }\end{array}$ & $\begin{array}{l}{[\ldots] \text { "La empresa identificó una necesidad sobre un nuevo producto, }} \\
\text { que fueron las bolas de Molino, que no comercializábamos y que se vio } \\
\text { que las mineras lo utilizan con frecuencia" (Industrias básica de hierro } \\
\text { y acero, empresa 12) }\end{array}$ \\
\hline $\begin{array}{l}\text { Innovación } \\
\text { en proceso }\end{array}$ & $\begin{array}{l}{[\ldots] \text { "Después de un análisis exhaustivo acerca de cada proceso se pudo }} \\
\text { modificar ciertos pasos con la finalidad de que se optimizaran tiempos } \\
\text { y las mermas disminuyan de forma significativa" (Elaboración y con- } \\
\text { servación de carne, empresa 1) }\end{array}$ \\
\hline
\end{tabular}

\subsection{Consecuencias}

El desarrollo de la capacidad de innovación de la empresa tiene como consecuencia la implementación de innovaciones tecnológicas y no tecnológicas.

\section{- Empresas de alimentos (baja intensidad tecnológica)}

La empresa 1 realizó innovación en procesos con la finalidad de reducir tiempos y mermas Además, implementa innovación en el área de comercialización para mejorar las estrategias que les permita incrementar las ventas. 


\section{- Empresas textiles (baja intensidad tecnológica)}

La empresa 5 realiza adquisiciones de máquinas de producción y equipo de laboratorio para mejorar la formulación de recetas para el teñido de tejidos. Así mismo, la empresa siempre mejora la relación con sus clientes.

- Empresas de productos de plástico o caucho

La empresa 8 ha realizado el lanzamiento de dos nuevos productos y se encuentran registrando dos nuevas patentes. También, mediante la adquisición de software, ha podido realizar mejoras en los procesos productivos.

- Empresas de fabricación de partes de vehículo

La empresa 9 ha ingresado a una nueva línea de producción que consiste en la fabricación de repuestos para camiones. En las innovaciones no tecnológicas, ha implementado una página web que le permite estar en contacto con sus clientes durante 24 horas al día y 7 días a la semana.

- Empresa de fabricación de maquinaria

La empresa 16 tiene planeado lanzar un nuevo tipo de maquinaria llamada Scoop, que mejora la línea de equipos de perforación de rocas. También, en la innovación por procesos, implementó un estudio de tiempos y movimientos, y compró un instrumento de torqueo regulable, que es utilizado por un solo operario y reduce los tiempos de montaje de 5 horas a casi 8 minutos.

\subsection{Covarianzas}

En la medida en que las empresas desarrollan mayor capacidad de innovación, tienen mayor predisposición a implementar innovaciones en productos y procesos. Así se puede afirmar que, a mayor capacidad de innovación, mayor número de innovaciones.

\subsection{Contingencias}

Las empresas peruanas de manufactura enfrentan contingencias particulares para el desempeño de sus actividades. Así, el Ministerio de la Producción (2016), al analizar la encuesta nacional de innovación de la industria de manufactura en el Perú de 2015, encontró que la mayoría de las empresas encuestadas son pequeñas y medianas, y presentan una intensidad tecnológica baja y media-baja.

\section{Discusiones y conclusiones}

Los hallazgos se pueden dividir en relación a dos aspectos: los factores internos y los factores externos, que están asociados a la capacidad de innovación de la empresa. En relación a los factores internos, se empezará señalando que el diseño y la aplicación de técnicas de ingeniería industrial es una de las actividades más realizadas por las pequeñas empresas de baja intensidad tecnológica. El diseño es una actividad relevante en la industria del calzado, en especial en la elaboración de nuevos modelos (innovación en productos), o en la industria de fibras textiles, en la que, aplicando 
técnicas de mejora continua, se logra reducir tiempos y costos, lo cual conlleva a la mejora del desempeño de la empresa.

La siguiente actividad más realizada es la adquisición de maquinaria, que es muy frecuente en las empresas de baja intensidad tecnológica. Las empresas pequeñas la realizan porque, de esta manera, logran introducir innovación en procesos. A las empresas medianas les permite ingresar a nuevos mercados con productos mejorados. Este resultado se condice con lo señalado por Tello (2017), quien indica que la adquisición de la maquinaria permite a las empresas mejorar su capacidad de innovación e implementar innovaciones en productos y procesos.

En relación a las empresas que obtienen certificación y control de calidad, se puede apreciar que, en su mayoría, son empresas grandes y presentan media-baja intensidad tecnológica. Estas prácticas de control de calidad llevan a las empresas a desarrollar innovación en procesos y en otros casos a implementar «sistemas ERP», lo cual lleva a implementar innovaciones organizacionales. Esto coincide con el punto de vista examinado por Bourke \& Roper (2017), quienes arguyen que la consistencia de la calidad de los productos generará más satisfacción en los clientes.

Por otro lado, Papa et al. (2018) señalan que la capacitación al personal mejora la capacidad de innovación de la empresa, lo cual concuerda con los resultados señalados por los entrevistados en las empresas pequeñas y grandes, tanto en las empresas de baja y baja-media intensidad tecnológica. Ellos manifiestan también que la capacitación al personal permite que éste conozca mejor la operación de las máquinas, así como el desarrollo de habilidades que fomentarán la innovación.

Analizando los hallazgos para los factores externos, se encontró que uno de los factores que más influye en el desarrollo de la capacidad de innovación de las empresas es su relación con los clientes o consumidores. Esto se aprecia tanto en las empresas pequeñas y grandes de baja intensidad. Los clientes proporcionan valiosa información para mejorar los productos y los procesos. Este resultado está en línea por lo señalado por Saldanha et al. (2017), quienes afirman que el consumidor ayuda a mejorar la capacidad de innovación de la empresa.

El siguiente factor externo es la relación o vinculación con los proveedores, que les permite a las pequeñas y grandes de baja y media-baja intensidad tecnológica mejorar su capacidad de innovación de la empresa. Se aprecia que las pequeñas empresas de baja intensidad tecnológica obtienen valiosa información de sus proveedores, que las conduce a realizar una mejora continua, en tanto que las empresas medianas de media-baja intensidad tecnológica visitan las ferias de proveedores para mejorar sus productos y procesos. Los proveedores constituyen una fuente importante de conocimiento, como también lo mencionan Roper et al. (2017).

De manera similar, la competencia ayuda a las empresas grandes y a las de baja intensidad tecnológica a mejorar su capacidad de innovación. Las empresas de baja intensidad tecnológica consideran a los competidores como un referente para mejorar sus propias estrategias de ventas y las empresas medianas, como aliciente para innovar. Este resultado concuerda por lo señalado por Qian \& Wang (2017), quienes arguyen que la competencia del mercado obliga a las empresas a innovar, y de esta manera diferenciarse de los competidores. 
También los consultores externos constituyen una valiosa fuente de conocimiento para las empresas pequeñas y de baja intensidad tecnológica. Las empresas grandes que tienen mayores recursos contratan consultores externos para desarrollar actividades de investigación y desarrollo, en tanto que las pequeñas empresas contratan consultores externos para que les orienten en la mejora de los procesos de fabricación y administrativos. En esa medida, nuestros hallazgos están alineados con los obtenidos por Musioliket et al. (2018).

El presente estudio contribuye a la literatura, pues se identifican los factores internos y externos que favorecen el desarrollo de la capacidad de innovación, según su tamaño e intensidad tecnológica. El desarrollo de actividades internas de innovación, tales como diseño y aplicación de técnicas de ingeniería industrial, la adquisición de maquinaria, la obtención de certificados de calidad y aplicación de técnicas de control de calidad, así como la capacitación del personal, permite el desarrollo de la capacidad de innovación de las empresas y, de esta manera, la implementación de innovaciones en productos y en procesos. Las empresas de manufactura se vinculan con los clientes o consumidores, proveedores, competidores y consultores externos para mejorar su capacidad de innovación. En la mayoría de los casos, las vinculaciones generan información o conocimiento que ayudan a las empresas a desarrollar innovaciones en productos o procesos y, en otros, les proporcionan el conocimiento para llevar a cabo actividades de investigación y desarrollo que contribuyen a mejorar el desempeño de sus empresas.

Se pueden señalar tres limitaciones principales: En primer lugar, resulta difícil decidir si se ha alcanzado la saturación teórica. En esta oportunidad, se ha logrado entrevistar a 16 personas que laboran en empresas de distintas industrias, de distintos tamaños, o que muestran tres de las cuatro intensidades tecnológica. Sin embargo, no se logró entrevistar a alguna empresa que presente alta intensidad tecnológica. En segundo lugar, la forma en la que se ha seleccionado la muestra también es una limitación, ya que se eligió a los participantes tomando en cuenta a las distintas industrias (rubros) a las que pertenecían o los distintos tamaños de las empresas, y según la facilidad para poder entrevistarlos. En tercer lugar, la información recolectada es la que fue proporcionada por los entrevistados. No se tuvo la oportunidad de observar directamente las relaciones que se establecían entre las empresas y sus proveedores o clientes.

A pesar de que el presente estudio tiene limitaciones, también es un aporte para la literatura de la innovación de las empresas de manufactura en una economía emergente, debido a que abre líneas de futuras investigaciones. Se recomienda que se analicen las innovaciones organizacionales y de comercialización, y cómo estas influyen en las innovaciones en producto y en proceso. También sería interesante analizar cómo las innovaciones están relacionadas con el mejor desempeño de la empresa o analizar una sola industria, teniendo en cuenta que, dentro ella, se pueden presentar comportamientos heterogéneos, que dependen del tipo de producto que se ofrece al mercado.

\section{Agradecimientos y apoyo}

Grupo de investigación: Innovación y emprendimiento, financiamiento interno del Vicerrectorado de Investigación de la Universidad ESAN, Lima, Perú. 


\section{Referencias}

Albis, N., \& Alvarez, I. (2017). Un análisis comparado del desempeño innovador de las empresas extranjeras y nacionales en la industria manufacturera de Colombia. Globalización, Competitividad y Gobernabilidad de Georgetown/ Universia, 11(2). https://doi.org/10.3232/GCG.2017.V11.N2.01

Bourke, J., \& Roper, S. (2017). Innovation, quality management and learning: Short-term and longer-term effects. Research Policy, 46(8), 1505-1518. https://doi.org/10.1016/j.respol.2017.07.005

Brenes, E. R., Camacho, A. R., Ciravegna, L., \& Pichardo, C. A. (2016). Strategy and innovation in emerging economies after the end of the commodity boom-Insights from Latin America. Journal of Business Research, 69(10), 4363-4367. https://doi.org/10.1016/j.jbusres.2016.03.059

Castellacci, F., \& Natera, J. M. (2012). Innovation surveys in Latin America: a primer. Innovation and Development, 2(1), 199-204. https://doi.org/10.1080/2157930x.2012.663585

Dunne, C. (2011). The place of the literature review in grounded theory research. International Journal of Social Research Methodology, 14(2), 111-124. https://doi.org/10.1080/13645579.201 0.494930

Ferraris, A., Santoro, G., \& Dezi, L. (2017). How MNC's subsidiaries may improve their innovative performance? The role of external sources and knowledge management capabilities. Journal of Knowledge Management, 21(3), 540-552. https://doi.org/10.1108/jkm-09-2016-0411

Ferreira de Lara, F., \& Neves Guimarães, M. R. (2014). Competitive priorities and innovation in SMEs: A Brazil multi-case study. Journal of technology management $\mathcal{E}$ innovation, 9(3), 51-64. https://doi.org/10.4067/s0718-27242014000300004

Gandomani, T. J., Zulzalil, H., Ghani, A. A. A., Sultan, A. B. M., \& Parizi, R. M. (2015). The impact of inadequate and dysfunctional training on Agile transformation process: a Grounded Theory study. Information and Software Technology, 57, 295-309. https://doi.org/10.1016/j. infsof.2014.05.011

Gandomani, T. J., Zulzalil, H., Ghani, A. A. A., Sultan, A. B. M., \& Sharif, K. Y. (2013). Exploring key factors of pilot projects in agile transformation process using a grounded theory study. International Conference on Information and Software Technologies (pp. 146-158). Springer, Berlin, Heidelberg. https://doi.org/10.1007/978-3-642-41947-8_14

Heredia, J., Flores, A., Geldes, C., \& Heredia, W. (2017). Effects of informal competition on innovation performance: the case of pacific alliance. Journal of Technology Management $\mathcal{E}$ Innovation, 12(4), 22-28. https://doi.org/10.4067/s0718-27242017000400003

Herman, H., Hady, H., \& Arafah, W. (2018). The Influence of Market Orientation and Product Innovation on the Competitive Advantage and Its Implication toward Small and Medium Enterprises (UKM) Performance. International Journal of Science and Engineering Invention, 4(08), 08-to. https://doi.org/10.23958/ijsei/vol04-i08/02

M. S. S., Kannan, V. R., Brah, S. A., \& Hassan, S. Z. (2017). Linkages between firm innovation strategy, suppliers, product innovation, and business performance: insights from resource dependence theory. International Journal of Operations $\mathcal{E}$ Production Management, 37(8), 10541075. https://doi.org/10.1108/ijopm-09-2014-0424

Ketata, I., Sofka, W., \& Grimpe, C. (2015). The role of internal capabilities and firms' environment for sustainable innovation: evidence for Germany. RED Management, 45(1), 60-75 https://doi.org/10.1111/radm.12052

Kim, M. K., Park, J. H., \& Paik, J. H. (2018). Factors influencing innovation capability of sma$\mathrm{ll}$ and medium-sized enterprises in Korean manufacturing sector: facilitators, barriers and moderators. International Journal of Technology Management, 76(3-4), 214-235. https://doi. org/10.1504/ijtm.2018.10012461

Lee, V. H., Leong, L. Y., Hew, T. S., \& Ooi, K. B. (2013). Knowledge management: a key determinant in advancing technological innovation. Journal of Knowledge Management, 17(6), 848872. https://doi.org/10.1108/jkm-08-2013-0315 
Liu, W., \& Atuahene-Gima, K. (2018). Enhancing product innovation performance in a dysfunctional competitive environment: The roles of competitive strategies and market-based assets. Industrial Marketing Management, 73, 7-20. https://doi.org/10.1016/j.indmarman.2018.01.006

Martínez-Costa, M., Jimenez-Jimenez, D., \& Castro-del-Rosario, Y. D. P. (2018). The performance implications of the UNE 166.000 standardised innovation management system. European Journal of Innovation Management. https://doi.org/10.1108/ejim-02-2018-0028

Ministerio de la Producción. (2016). Estudio de la situación actual de la innovación en la industria manufacturera. Análisis de los resultados de la Encuesta Nacional de Innovación de la Industria Manufacturera 2015.

Musiolik, J., Markard, J., Hekkert, M., \& Furrer, B. (2018). Creating innovation systems: How resource constellations affect the strategies of system builders. Technological Forecasting and Social Change (In Press). https://doi.org/10.1016/j.techfore.2018.02.002

Olavarrieta, S., \& Villena, M. G. (2014). Innovation and business research in Latin America: An overview. Journal of Business Research, 67(4), 489-497. https://doi.org/10.1016/j.jbusres.2013.11.005

Organization for Economic Cooperation and Development (OECD) (2007). Science, Technology and Industry Scoreboard, OECD, Paris. https://doi.org/10.1787/sti_scoreboard-2007-en

Papa, A., Dezi, L., Gregori, G. L., Mueller, J., \& Miglietta, N. (2018). Improving innovation performance through knowledge acquisition: the moderating role of employee retention and human resource management practices. Journal of Knowledge Management. https://doi. org/10.1108/JKM-09-2017-0391

Paunov, C. (2016). Corruption's asymmetric impacts on firm innovation. Journal of Development Economics, 118, 216-231. https://doi.org/10.1016/j.jdeveco.2015.07.006

Pérez, J. A. H., Geldes, C., Kunc, M. H., \& Flores, A. (2018). New approach to the innovation process in emerging economies: The manufacturing sector case in Chile and Peru. Technovation, 79, 35-75. https://doi.org/10.1016/j.technovation.2018.02.012

Qian, L., \& Wang, I. K. (2017). Competition and innovation: The tango of the market and technology in the competitive landscape. Managerial and Decision Economics, 38(8), 1237-1247. https://doi.org/10.1002/mde.2861

Roper, S., Love, J. H., \& Bonner, K. (2017). Firms' knowledge search and local knowledge externalities in innovation performance. Research Policy, 46(1), 43-56. https://doi.org/10.1016/j. respol.2016.10.004

Saldanha, T. J., Mithas, S., \& Krishnan, M. S. (2017). Leveraging Customer Involvement for Fueling Innovation: The Role of Relational and Analytical Information Processing Capabilities. MIS Quarterly, 41(1). https://doi.org/10.25300/misq/2017/41.1.14

Sanz, L., \& Jones, V. (2013). Advances in business research in Latin American studies. Journal of Business Research, 66(3), 397-400. https://doi.org/10.1016/j.jbusres.2012.04.005

Simeone, L., Secundo, G., \& Schiuma, G. (2017). Knowledge translation mechanisms in open innovation: the role of design in R\&D projects. Journal of Knowledge Management, 21(6), 14061429. https://doi.org/10.1108/jkm-10-2016-0432

Scott, G. J., \& Chaston, I. (2012). Culture and innovation in Peru from a management perspective. Journal of Global Initiatives, 7(2), 131-145. https://doi.org/10.7835/ccwp-2012-09-0010

Strauss, A., \& Corbin, J. (1994). Grounded Theory Methodology: An Overview. In N. K. Denzin, \& Y. S. Lincoln (Eds.), Handbook of Qualitative Research (Charpter 17, pp. 273-285). Thousand Oaks, CA: SAGE.

Tello, M. D. (2017). Innovation and productivity in services and manufacturing firms: the case of Peru. CEPAL Review. https://doi.org/10.18356/a4c7eea5-en

Zawislak, P. A., Fracasso, E. M., \& Tello-Gamarra, J. (2018). Technological intensity and innovation capability in industrial firms. Innovation $\mathcal{E}$ Management Review, 15(2), 189-207. https:// doi.org/10.1108/inmr-04-2018-012 